\title{
Pairing Scenarios for the Hubbard Model in the Strong Coupling Limit
}

\author{
V.A. Apinyan And T.K. Kopeć \\ Institute for Low Temperature and Structure Research \\ P.O. Box 1410, 50-950 Wrocław 2, Poland
}

\begin{abstract}
We consider a spin-rotationally invariant Hubbard model. The original electron operator is presented in the charge-spin-fermion $\mathrm{U}(1) \times \mathrm{SU}(2)$ factorized form. The factorization procedure is given in terms of the emergent gauge fields. As a result, the electron appears like a composite object consisting of strongly fluctuating phase field and spatially rotating spin axis. Furthermore, we elaborate microscopically on the form of possible pairing states.

PACS numbers: 71.27.+a, 71.10.Fd, 74.20.Rp
\end{abstract}

\section{Introduction}

The Hubbard Hamiltonian is considered as a most purposeful one for studying the strongly interacting electronic systems. It contains the essential background in the physics of the interacting electrons. We consider the Hubbard Hamiltonian in the spin-rotational invariant form [1]. To keep the spin-rotationally invariance we rewrite the action of the system transforming it into new bosonic and fermionic variables. The relevant transformations are based on the spin-charge separation that allows the electrons to behave as a bound state of two independent components, spinon and chargon. Using the $\mathrm{U}(1)$ and $\mathrm{SU}(2)$ transformations we factorize the electron operator in terms of the emergent gauge fields. Using the functional integration formalism [2] we construct the grand canonical partition function of the system. The effective fermionic action will be generated by cumulant expansion. To look for a pairing interaction we concentrate on the second-order cumulant containing the four fermion operators [3]. With connection to the spin-fermionic part we derive microscopically the final form of the action in both nearest neighbors (n.n.) and next nearest neighbors (n.n.n.) cases. After the Schwinger boson transformations it is obvious that in the nearest neighbors case there is no evidence for pairing interactions. For the next nearest neighbors case there is a possibility for singlet or triplet pairing state. The corresponding pairing gaps are expressed as a function of bosonic $\mathrm{CP}^{1}$ Green functions and spin-fermionic part appearing in the form of the Schwinger-boson and Schwinger-fermion valence bond operators, respectively. 


\section{The second-order cumulant and angular-bosonic correlator}

Our Hamiltonian of the Hubbard model is $H=H_{t}+H_{t^{\prime}}+H_{U}$, where

$$
H_{t}+H_{t^{\prime}}=-\sum_{\alpha} \sum_{\boldsymbol{r} \boldsymbol{r}^{\prime}}\left(t \delta_{\boldsymbol{r}-\boldsymbol{r}^{\prime}, \boldsymbol{d}}+t^{\prime} \delta_{\boldsymbol{r}-\boldsymbol{r}^{\prime}, \boldsymbol{d}^{\prime}}\right)\left[c_{\alpha}^{\dagger}(\boldsymbol{r} \tau) c_{\alpha}\left(\boldsymbol{r}^{\prime} \tau\right)+\text { h.c. }\right]
$$

and $H_{U}=\sum_{\boldsymbol{r}} U n_{\uparrow}(\boldsymbol{r}) n_{\downarrow}(\boldsymbol{r})$. The vectors $\boldsymbol{d}$ and $\boldsymbol{d}^{\prime}$ are the lattice vectors for n.n. and n.n.n. cases, respectively, and $U$ stands for the Coulomb repulsion. Following [3] we rewrite the interaction term in the spin-rotational invariant form $H_{U}=U \sum_{\boldsymbol{r}}\left\{\frac{1}{4} n^{2}(\boldsymbol{r} \tau)-[\boldsymbol{\Omega}(\boldsymbol{r} \tau) \cdot \boldsymbol{S}(\boldsymbol{r} \tau)]^{2}\right\}$, where $\boldsymbol{\Omega}$ is the unit vector and $S_{\mathrm{c}}^{a}(\boldsymbol{r} \tau)=\frac{1}{2} \sum_{\alpha \beta} c_{\alpha}^{\dagger}(\boldsymbol{r} \tau) \hat{\sigma}_{\alpha \beta}^{a} c_{\beta}(\boldsymbol{r} \tau)$ is the spin operator $(a=x, y, z)$ with $\hat{\sigma}^{a}$ being the Pauli matrices. The spin-charge decomposition is given by $c_{\alpha}(\boldsymbol{r} \tau)=\sum_{\beta} z(\boldsymbol{r} \tau) \boldsymbol{R}_{\alpha \beta}(\boldsymbol{r} \tau) h_{\beta}(\boldsymbol{r} \tau)$, where $z(\boldsymbol{r} \tau)$ is the new unimodular phase $\mathrm{U}(1)$ variable [3, 4], $\boldsymbol{R}_{\alpha \beta}(\boldsymbol{r} \tau)$ is the spin-rotation $\mathrm{SU}(2)$ matrix [4] and $h_{\alpha}(\boldsymbol{r} \tau)$ are new fermionic variables [3]. With $\mathrm{U}(1)$ and $\mathrm{SU}(2)$ transformations we unraveled the electron operator to a composite object consisting of fermions attached to the fluctuating gauge potentials. The $\mathrm{SU}(2)$ transformation is done by means of the Hopf map $\boldsymbol{R}(\boldsymbol{r} \tau) \hat{\sigma}^{z} \boldsymbol{R}^{\dagger}(\boldsymbol{r} \tau)=\hat{\sigma} \boldsymbol{\Omega}$. U(1) and $\mathrm{SU}(2)$ gauge fields play a specific role in the formulated problem. They are responsible for the electron pairing similar to the phonons in the BCS superconductors. Regarding the Hubbard Hamiltonian it is evident that these fields, the collective high energy modes in the superconducting (SC) system, couple to the fermion density type term via the hopping amplitude $t$ [3]. We evaluate the effective interaction between fermions by tracing out the gauge degrees of freedom. We write the partition function as $Z=\int[D \bar{h} D h] \mathrm{e}^{-S_{\text {eff }}[\bar{h}, h]}$, where we have integrated over the charge and angular variables. The effective action in the exponential is $S_{\text {eff }}[\bar{h}, h]=-\ln \int[D \boldsymbol{\Omega} D \phi] \mathrm{e}^{-S[\Omega, \phi, \bar{h}, h]}[3]$. We consider the second order cumulant generated from the effective action. After averaging over the charge $\mathrm{U}(1)$ field we obtain

$$
\begin{aligned}
S^{(2)}[\bar{h}, h]=S_{1}^{(2)}[\bar{h}, h]+S_{2}^{(2)}[\bar{h}, h] \\
=-\frac{t^{2}}{U} \sum_{\left\langle\boldsymbol{r} \boldsymbol{r}^{\prime}\right\rangle} \sum_{\alpha \alpha^{\prime}, \gamma \gamma^{\prime}} \int_{0}^{\beta} \mathrm{d} \tau\left\langle\boldsymbol{M}_{\alpha^{\prime} \alpha, \gamma \gamma^{\prime}}\right\rangle \bar{h}_{\alpha^{\prime}}(\boldsymbol{r} \tau) h_{\alpha}\left(\boldsymbol{r}^{\prime} \tau\right) \bar{h}_{\gamma}\left(\boldsymbol{r}^{\prime} \tau\right) h_{\gamma^{\prime}}(\boldsymbol{r} \tau) \\
\quad-\frac{t^{\prime 2}}{U} \sum_{\left\langle\left\langle\boldsymbol{r} \boldsymbol{r}^{\prime}\right\rangle\right\rangle} \sum_{\alpha \alpha^{\prime}, \gamma \gamma^{\prime}} \int_{0}^{\beta} \mathrm{d} \tau\left\langle\boldsymbol{M}_{\alpha^{\prime} \alpha, \gamma \gamma^{\prime}}^{\prime}\right\rangle \bar{h}_{\alpha^{\prime}}(\boldsymbol{r} \tau) h_{\alpha}\left(\boldsymbol{r}^{\prime} \tau\right) \bar{h}_{\gamma}\left(\boldsymbol{r}^{\prime} \tau\right) h_{\gamma^{\prime}}(\boldsymbol{r} \tau),
\end{aligned}
$$

where $\boldsymbol{M}_{\alpha^{\prime} \alpha, \gamma \gamma^{\prime}}\left(\boldsymbol{r} \tau, \boldsymbol{r}^{\prime} \tau \mid \boldsymbol{r}^{\prime} \tau, \boldsymbol{r} \tau\right)=\left[\boldsymbol{R}^{\dagger}(\boldsymbol{r} \tau) \boldsymbol{R}\left(\boldsymbol{r}^{\prime} \tau\right)\right]_{\alpha^{\prime} \alpha}\left[\boldsymbol{R}^{\dagger}\left(\boldsymbol{r}^{\prime} \tau\right) \boldsymbol{R}(\boldsymbol{r} \tau)\right]_{\gamma \gamma^{\prime}}$ and $\langle\ldots\rangle$ denotes averaging over $\mathrm{U}(1)$ and $\mathrm{SU}(2)$ fields. We define the $\mathrm{SU}(2)$ matrix $\boldsymbol{R}(\boldsymbol{r} \tau)$ in the complex projective field

$$
\boldsymbol{R}(\boldsymbol{r} \tau)=\left[\begin{array}{cc}
\zeta_{1}(\boldsymbol{r} \tau) & -\bar{\zeta}_{2}(\boldsymbol{r} \tau) \\
\zeta_{2}(\boldsymbol{r} \tau) & \bar{\zeta}_{1}(\boldsymbol{r} \tau)
\end{array}\right]
$$

where $\zeta(\boldsymbol{r} \tau)$ are complex $\mathrm{CP}^{1}$ variables. They are satisfying: $\left|\zeta_{1}(\boldsymbol{r} \tau)\right|^{2}+$ $\left|\zeta_{2}(\boldsymbol{r} \tau)\right|^{2}=1$. 


\section{The Schwinger transformation}

In this section we introduce the antiferromagnetic Schwinger boson AFM-B valence bond operators (see, for instance $\left.\left[\begin{array}{ll}5, & 6\end{array}\right]\right) \mathcal{F}\left(\boldsymbol{r} \tau \boldsymbol{r}^{\prime} \tau\right)=$ $\bar{\zeta}_{1}(\boldsymbol{r} \tau) \zeta_{1}\left(\boldsymbol{r}^{\prime} \tau\right)+\bar{\zeta}_{2}(\boldsymbol{r} \tau) \zeta_{2}\left(\boldsymbol{r}^{\prime} \tau\right)$ and $\mathcal{A}\left(\boldsymbol{r} \tau \boldsymbol{r}^{\prime} \tau\right)=\zeta_{1}(\boldsymbol{r} \tau) \zeta_{2}\left(\boldsymbol{r}^{\prime} \tau\right)-\zeta_{2}(\boldsymbol{r} \tau) \zeta_{1}\left(\boldsymbol{r}^{\prime} \tau\right)$. Then, the product of two SU(2) matrices can be written in the composed form

$$
\boldsymbol{R}^{\dagger}(\boldsymbol{r} \tau) \boldsymbol{R}\left(\boldsymbol{r}^{\prime} \tau\right)=\left[\begin{array}{rr}
\mathcal{F} & -\overline{\mathcal{A}} \\
\mathcal{A} & \overline{\mathcal{F}}
\end{array}\right]
$$

Consequently, the matrix $\hat{\boldsymbol{M}}$ takes the following form:

$$
\hat{\boldsymbol{M}}\left(\boldsymbol{r} \tau, \boldsymbol{r}^{\prime} \tau \mid \boldsymbol{r}^{\prime} \tau, \boldsymbol{r} \tau\right)=\left[\begin{array}{rrrr}
\mathcal{F} \overline{\mathcal{F}} & \mathcal{F} \overline{\mathcal{A}} & -\mathcal{F} \mathcal{A} & \mathcal{F} \mathcal{F} \\
-\overline{\mathcal{A}} \overline{\mathcal{F}} & -\overline{\mathcal{A}} \overline{\mathcal{A}} & \overline{\mathcal{A}} \mathcal{A} & -\overline{\mathcal{A}} \mathcal{F} \\
\mathcal{A} \overline{\mathcal{F}} & \mathcal{A} \overline{\mathcal{A}} & -\mathcal{A} \mathcal{A} & \mathcal{A} \mathcal{F} \\
\overline{\mathcal{F}} \overline{\mathcal{F}} & \overline{\mathcal{F}} \overline{\mathcal{A}} & -\overline{\mathcal{F}} \mathcal{A} & \overline{\mathcal{F}} \mathcal{F}
\end{array}\right] .
$$

The saddle-point evaluations for the external potential, coupled to the fermions via the Zeeman-type term [3], give a staggering charge-gap parameter and our lattice is a cross-match of two fundamental ferromagnetic sublattices. In particular, $\left\langle S_{\mathrm{h}}^{z}(\boldsymbol{r})\right\rangle=(-1)^{\boldsymbol{r}} \Delta_{\mathrm{c}}$, where $\Delta_{\mathrm{c}}$ is the charge gap and $(-1)^{\boldsymbol{r}}=\mathrm{e}^{\mathrm{i} \boldsymbol{Q} \cdot \boldsymbol{r}}$. Here, $\boldsymbol{Q}$ is defined as having the value $\pi / a$ in all components and $\boldsymbol{r}$ is the position of the lattice site. We analyze the form of the fermionic action by applying the Schwinger-boson transformation to the $\boldsymbol{R}(\boldsymbol{r} \tau)$ matrix: $\boldsymbol{R}(\boldsymbol{r} \tau)=\left(\mathrm{i} \hat{\sigma}_{y}\right) \widetilde{\boldsymbol{R}}(\boldsymbol{r} \tau)$, where $\widetilde{\boldsymbol{R}}(\boldsymbol{r} \tau)$ is the transformed form of the rotation matrix. Thus the angular-bosonic correlator (5) takes the symmetrized form and can be easily connected to the fermionic part. For the nearest neighbors term we obtain

$$
S_{1}^{(2)}[\bar{h}, h]=\sum_{\left\langle\boldsymbol{r} \boldsymbol{r}^{\prime}\right\rangle} \int_{0}^{\beta} \mathrm{d} \tau\left[g_{1} N(\boldsymbol{r} \tau) N\left(\boldsymbol{r}^{\prime} \tau\right)-g_{2} \bar{T}\left(\boldsymbol{r} \tau \boldsymbol{r}^{\prime} \tau\right) T\left(\boldsymbol{r} \tau \boldsymbol{r}^{\prime} \tau\right)\right]
$$

where $N(\boldsymbol{r} \tau)=\sum_{\alpha} \bar{h}_{\alpha}(\boldsymbol{r} \tau) h_{\alpha}(\boldsymbol{r} \tau)$ is the particle number operator at the site $\boldsymbol{r}$, the operator $T\left(\boldsymbol{r} \tau \boldsymbol{r}^{\prime} \tau\right)$ is the antiferromagnetic fermion AFM-F bond operator defined as $T\left(\boldsymbol{r} \tau \boldsymbol{r}^{\prime} \tau\right)=\frac{1}{\sqrt{2}} \sum_{\alpha} h_{\alpha}(\boldsymbol{r} \tau) h_{\alpha}\left(\boldsymbol{r}^{\prime} \tau\right)$. The coupling coefficient $g_{1}$ in the first term of the action is positive: $g_{1}=\frac{2 t^{2}}{U}\left(f^{2}+2 g_{0}^{2}\right)>0$, and therefore we have the possibility for the charge density wave (CDW) order. The Green functions $f$ and $g_{0}$ figuring in the expression of the $g_{1}$ are defined in the $\mathrm{CP}^{1}$ representation as: $f=\left\langle\zeta(\boldsymbol{r} \tau) \zeta\left(\boldsymbol{r}^{\prime} \tau\right)\right\rangle$ and $g_{0}=-\langle\zeta(\boldsymbol{r} \tau) \bar{\zeta}(\boldsymbol{r} \tau)\rangle$. The second-order term in the action shows a possible spin-triplet correlation mechanism. That is evident because the coefficient $g_{2}>0$ and is given by $g_{2}=\frac{2 t^{2}}{U}\left(2 f^{2}+g_{0}^{2}\right)$. A simple Hartree-Fock (HF) decoupling of the four-fermion term in Eq. (6) gives the solution for the triplet fermionic gap parameter $\Delta^{t}\left(\boldsymbol{r} \tau \boldsymbol{r}^{\prime} \tau\right)=\left\langle\bar{T}\left(\boldsymbol{r} \tau \boldsymbol{r}^{\prime} \tau\right)\right\rangle$. On the other hand, the gap function is defined as $\Delta_{\alpha \beta}^{\mathrm{c}}\left(\boldsymbol{r} \tau \boldsymbol{r}^{\prime} \tau\right)=\left\langle\bar{c}_{\alpha}(\boldsymbol{r} \tau) \bar{c}_{\beta}\left(\boldsymbol{r}^{\prime} \tau\right)\right\rangle$. We consider all possible pairings: singlet $\Delta_{\uparrow \downarrow}^{\mathrm{c}}, \Delta_{\downarrow \uparrow}^{\mathrm{c}}$ and triplet $\Delta_{\uparrow \uparrow}^{\mathrm{c}}, \Delta_{\downarrow \downarrow}^{\mathrm{c}}$. In the nearest neighbors case, for the singlet gap function $\Delta_{\mathrm{S}} \equiv \Delta_{\uparrow \downarrow}^{\mathrm{c}}-\Delta_{\downarrow \uparrow}^{\mathrm{c}}$ we obtain $\Delta_{\mathrm{S}}=$ 
$-2 \sqrt{2} f\left\langle\bar{z}(\boldsymbol{r} \tau) \bar{z}\left(\boldsymbol{r}^{\prime} \tau\right)\right\rangle\left\langle\bar{T}\left(\boldsymbol{r} \tau \boldsymbol{r}^{\prime} \tau\right)\right\rangle$, where the complex variable $z(\boldsymbol{r} \tau)=\mathrm{e}^{\mathrm{i} \phi(\boldsymbol{r} \tau)}$ describe the charge parametrization with $|z(\boldsymbol{r} \tau)|^{2}=1$. As follows from definition, the function $\Delta_{\mathrm{S}}$ is even under spin reversion transformation while the operator $T$, in the right hand side, is odd. The second source for possible pairings is the action

$$
\begin{gathered}
S_{2}^{(2)}[\bar{h}, h]=\sum_{\left\langle\left\langle\boldsymbol{r} \boldsymbol{r}^{\prime}\right\rangle\right\rangle} \int_{0}^{\beta}\left[g_{1}^{\prime} N(\boldsymbol{r} \tau) N\left(\boldsymbol{r}^{\prime} \tau\right)-g_{2}^{\prime} \bar{F}\left(\boldsymbol{r} \tau \boldsymbol{r}^{\prime} \tau\right) F\left(\boldsymbol{r} \tau \boldsymbol{r}^{\prime} \tau\right)\right. \\
\left.-g_{2}^{\prime} \bar{A}\left(\boldsymbol{r} \tau \boldsymbol{r}^{\prime} \tau\right) A\left(\boldsymbol{r} \tau \boldsymbol{r}^{\prime} \tau\right)-g_{3}^{\prime} \bar{T}\left(\boldsymbol{r} \tau \boldsymbol{r}^{\prime} \tau\right) T\left(\boldsymbol{r} \tau \boldsymbol{r}^{\prime} \tau\right)\right] .
\end{gathered}
$$

As in the previous case the first term is responsible for CDW order in the system, because $g_{1}^{\prime}>0: g_{1}^{\prime}=\frac{2 t^{\prime 2}}{U}\left(\frac{1}{2} g^{2}+\frac{1}{2} f_{0}^{2}+g_{0}^{2}\right)$. Here, the Green function $g$ is defined as usual: $g=-\left\langle\zeta(\boldsymbol{r}) \bar{\zeta}\left(\boldsymbol{r}^{\prime}\right)\right\rangle$ and the anomalous on-site Green function $f_{0}$ is: $f_{0}=$ $\langle\zeta(\boldsymbol{r}) \zeta(\boldsymbol{r})\rangle$. In the second term the AFM-F Schwinger bond operator $F\left(\boldsymbol{r} \tau \boldsymbol{r}^{\prime} \tau\right)$ is defined as $F\left(\boldsymbol{r} \tau \boldsymbol{r}^{\prime} \tau\right)=\frac{1}{\sqrt{2}}\left[\bar{h}_{\uparrow}(\boldsymbol{r} \tau) h_{\uparrow}\left(\boldsymbol{r}^{\prime} \tau\right)+\bar{h}_{\downarrow}(\boldsymbol{r} \tau) h_{\downarrow}\left(\boldsymbol{r}^{\prime} \tau\right)\right]$. The coefficient $g_{2}^{\prime}$ is given by $g_{2}^{\prime}=\frac{2 t^{\prime 2}}{U}\left(3 g^{2}+f_{0}^{2}\right)$ and is positive, hence after mean field decoupling this term contributes to the kinetic energy of the electrons. From the action (7) we can obtain the spin-singlet superconducting electronic correlations because $g_{2}^{\prime}>0$. The HF decoupling of the third four fermion term gives the corresponding pairing gap parameter $\Delta^{\mathrm{s}}\left(\boldsymbol{r} \tau \boldsymbol{r}^{\prime} \tau\right)=\left\langle\bar{A}\left(\boldsymbol{r} \tau \boldsymbol{r}^{\prime} \tau\right)\right\rangle$, where we introduced the AFM-F bond operator $A\left(\boldsymbol{r} \tau \boldsymbol{r}^{\prime} \tau\right)$ as $A\left(\boldsymbol{r} \tau \boldsymbol{r}^{\prime} \tau\right)=\frac{1}{\sqrt{2}}\left[h_{\uparrow}(\boldsymbol{r} \tau) h_{\downarrow}\left(\boldsymbol{r}^{\prime} \tau\right)-h_{\downarrow}(\boldsymbol{r} \tau) h_{\uparrow}\left(\boldsymbol{r}^{\prime} \tau\right)\right]$. For the singlet order parameter we have $\Delta_{\mathrm{S}}=\Delta_{\uparrow \downarrow}^{\mathrm{c}}-\Delta_{\downarrow \uparrow}^{\mathrm{c}}=-2 \sqrt{2} g\left\langle\bar{z}(\boldsymbol{r} \tau) \bar{z}\left(\boldsymbol{r}^{\prime} \tau\right)\right\rangle\left\langle\bar{A}\left(\boldsymbol{r} \tau \boldsymbol{r}^{\prime} \tau\right)\right\rangle$. The form of the action (7) could be also in favor of the spin-triplet pairing interactions, if the coefficient $g_{3}^{\prime}>0: g_{3}^{\prime}$ is given by: $g_{3}^{\prime}=\frac{2 t^{\prime 2}}{U}\left(2 f_{0}^{2}-2 f^{2}\right)$, where the Green functions $g_{0}$ and $f$ are defined above. Detailed direct calculations of corresponding gap functions justify the existence of mentioned pairing correlations in the next nearest neighbors case. Spin-triplet correlations are $\Delta_{\mathrm{T}}^{(1)}=\Delta_{\uparrow \downarrow}^{\mathrm{c}}+\Delta_{\downarrow \uparrow}^{\mathrm{c}}=0$ and $\Delta_{\mathrm{T}}^{(2)}=\Delta_{\uparrow \uparrow}^{\mathrm{c}}+\Delta_{\downarrow \downarrow}^{\mathrm{c}}=-2 \sqrt{2} f\left\langle\bar{z}(\boldsymbol{r} \tau) \bar{z}\left(\boldsymbol{r}^{\prime} \tau\right)\right\rangle\left\langle\bar{T}\left(\boldsymbol{r} \tau \boldsymbol{r}^{\prime} \tau\right)\right\rangle$. With switching the long-order mechanism of correlation, the electronic system shows the pairing abilities and both singlet and triplet correlations become possible. Future detailed microscopic calculations [7] of correlation functions from functional derivation technics will show the straightness of the pairing interactions in competition with the charge density wave fluctuations in (7) and clarify the role of the dominating pair ordered regions in the scenario of the superconducting phase transition.

\section{Acknowledgments}

T.K.K. acknowledges the support by the Ministry of Education and Science MEN under grant No. 1PO3B 10330 in the years 2006-2008. V.A.A. acknowledges the support by the International Max Planck Research School in Dresden. 


\section{References}

[1] T.K. Kopec, Phys. Stat. Sol. B 244, 2458 (2007).

[2] See for instance J.W. Negele, H. Orland, in: Quantum Many Particle Systems, Frontiers in Physics, Addison-Wesley, Boston 1988.

[3] T.K. Kopec, Phys. Rev. B 73, 132512 (2006).

[4] N. Dupuis, S. Pairault, Int. J. Mod. Phys. B 14, 2529 (2000).

[5] G. Baskaran, Z. Zou, P.W. Anderson, Solid State Commun. 63, 973 (1987).

[6] A. Auerbach, Interacting Electrons and Quantum Magnetism, Springer-Verlag, New York 1994.

[7] V.A. Apinyan, T.K. Kopeć, in preparation. 